\title{
Successful treatment of large-size advanced hepatocellular carcinoma by transarterial chemoembolization followed by the combination therapy of percutaneous ethanol-lipiodol injection and radiofrequency ablation
}

\author{
KAZUTAKA KUROKOHCHI $^{1,2}$, NAOKI HOSOMI ${ }^{1}$, AKIRA YOSHITAKE ${ }^{1,2}$, TOMOHIRO OHGI $^{1,2}$, \\ MASAHIRO ONO ${ }^{1,2}$, TSUYOSHI MAETA ${ }^{2}$, TAKAAKI KIUCHI ${ }^{3}$, ICHIRO MATSUMOTO ${ }^{4}$, \\ TSUTOMU MASAKI $^{1}$, HIROHITO YONEYAMA ${ }^{1}$, FUMIKAZU KOHI ${ }^{2}$ and SHIGEKI KURIYAMA ${ }^{1}$ \\ ${ }^{1}$ Department of Gastroenterology and Neurology, Kagawa University School of Medicine, 1750-1 Ikenobe, Miki-cho, \\ Kita-gun, Kagawa 761-0793; Departments of ${ }^{2}$ Gastroenterology, ${ }^{3}$ Radiology and ${ }^{4}$ Cardiology, \\ KKR Takamatsu Hospital, 4-18 Tenjinmae, Takamatsu, Kagawa 760-0018, Japan
}

Received April 26, 2006; Accepted July 18, 2006

\begin{abstract}
We report a case of large-size hepatocellular carcinoma (HCC) successfully treated with transarterial chemoembolization (TACE) followed by the combination therapy of percutaneous ethanol-lipiodol injection and radiofrequency ablation (PELI-RFA) and percutaneous ethanol-lipiodol injection (PELI) therapy. In the present case, the patient had a large-size advanced $\mathrm{HCC}, 7 \mathrm{~cm}$ in diameter, located in the S8 region of the liver. In addition, the hepatic reserve of the patient was severely poor. In order not to impair the poor hepatic reserve, we chose PELI-RFA and PELI, originally developed in our department and reported as milder treatment modalities than others. After TACE , PELIRFA and PELI were performed several times, the HCC was totally destroyed and early enhancement shown by helical dynamic computed tomography disappeared completely after treatment. The hepatic reserve of the patient was not impaired by the series of treatments. Serum levels of tumor markers, $\alpha$-fetoprotein and Des- $\gamma$-carboxy prothrombin, were rapidly decreased to almost normal levels. PELI-RFA and PELI may be effective for the treatment of large-size HCC of patients with poor hepatic reserve.
\end{abstract}

\section{Introduction}

Hepatocellular carcinoma (HCC) is still one of the most progressive malignancies resistant to treatment (1). Repeated

Correspondence to: Dr Shigeki Kuriyama, Department of Gastroenterology and Neurology, Kagawa University School of Medicine, 1750-1 Ikenobe, Miki-cho, Kita-gun, Kagawa 761-0793, Japan

E-mail: skuriyam@med.kagawa-u.ac.jp

Key words: hepatocellular carcinoma, ethanol-lipiodol injection, radiofrequency ablation, transarterial chemoembolization recurrences often occur due to multicentric carcinogenesis and intrahepatic metastasis. Although several treatment modalities are currently available for HCC, large-size advanced HCC is still difficult to treat. Among therapies, radiofrequency ablation (RFA) now plays a central role for the local control of hepatic malignancies (2-4). However, several limitations of RFA have been pointed out, such as limited coagulated necrosis and relatively frequent local tumor recurrences (5). To overcome these limitations, we have developed several percutaneous treatments. We showed that the combination therapy of percutaneous ethanol injection and RFA (PEI-RFA) could induce wider coagulated necrosis with a smaller energy requirement compared to routine RFA $(6,7)$. Furthermore, we also developed a percutaneous treatment of injecting a mixture of ethanol and lipiodol (percutaneous ethanol-lipiodol injection therapy, PELI) instead of ethanol alone $(8,9)$. Recently, we experienced a case of large-size advanced HCC formed on the cirrhotic liver with poor hepatic reserve which was successfully treated with PELI-RFA and PELI without impairing hepatic reserve.

\section{Case report}

A 68-year-old female attended a health examination, and a large-size tumor was found in the right hepatic lobe by ultrasonography (US). She was referred to our hospital for diagnosis and treatment of the tumor. By US, the large-size tumor was visualized as a round-shaped low-echoic lesion with a halo (Fig. 1A), and a daughter nodule of $2.5 \mathrm{~cm}$ in diameter was also detected (Fig. 1B). Helical dynamic computed tomography (CT) showed enhancement of the lesion in the early vascular phase and defect in the late phase (Fig. 2). The patient was positive for hepatitis $\mathrm{C}$ virus antibody and had high serum levels of $\alpha$-fetoprotein (AFP) and Des- $\gamma$ carboxy prothrombin (PIVKA II) (AFP, $127 \mathrm{ng} / \mathrm{ml}$; PIVKA II, $8150 \mathrm{MAU} / \mathrm{ml}$ ). Based on these findings, the tumors were easily diagnosed as HCC. A chest X-ray examination revealed that the large-size tumor was protruding from the surface of 


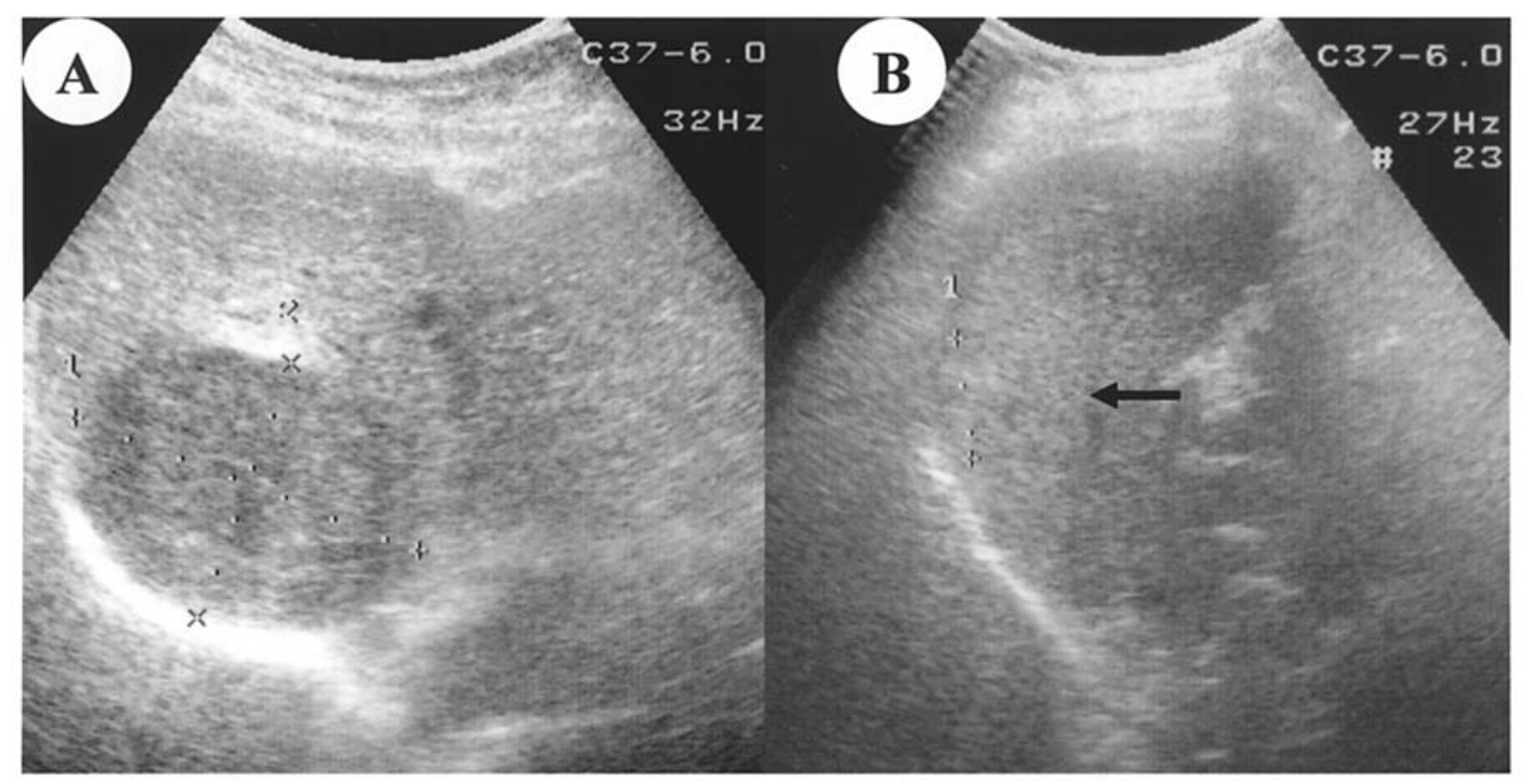

Figure 1. US images of the large-size HCC located in the S8 region of the liver immediately under the diaphragm (A) and the daughter nodule beside the large-size tumor (arrow) (B). The tumor was visualized from the bottom of the right costal arch. The large-size tumor was approximately $70 x 50 \mathrm{~mm}$ in diameter.

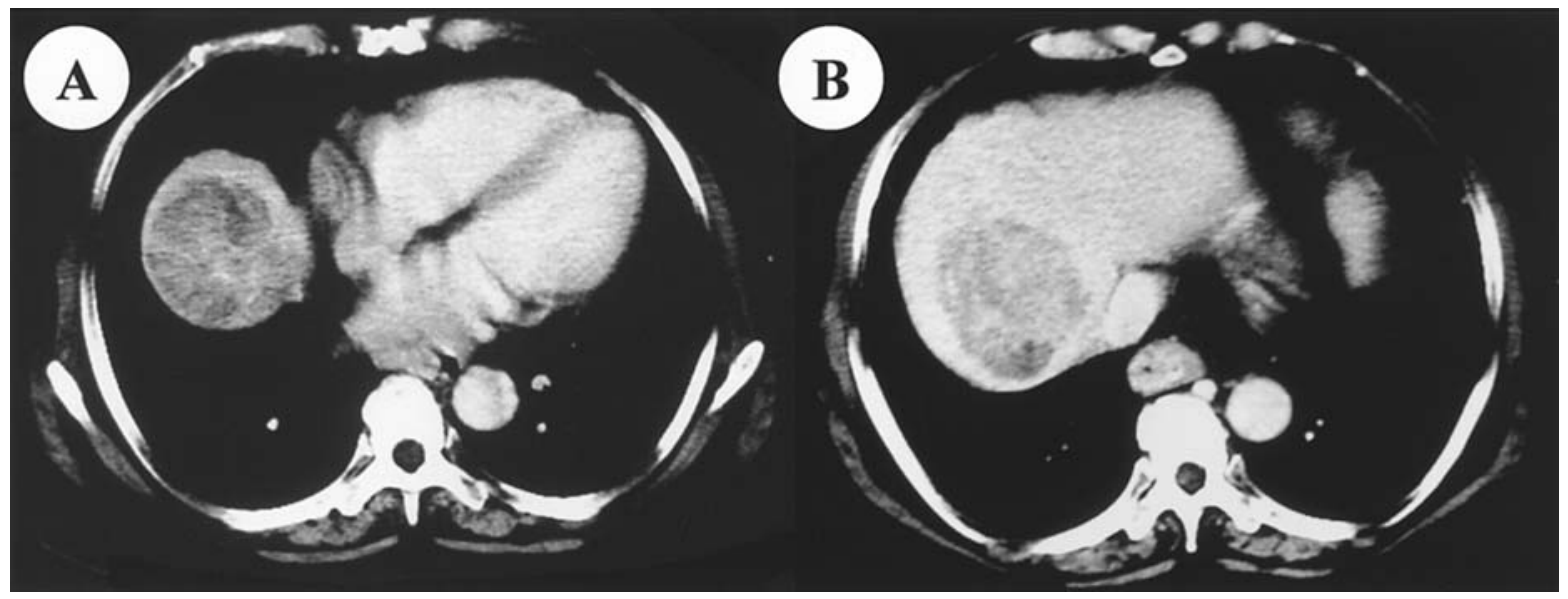

Figure 2. Helical dynamic CT images of the large-size tumor in the S8 region of the liver. CT images in the early vascular phase (A) and in the late phase (B) of the tumor.

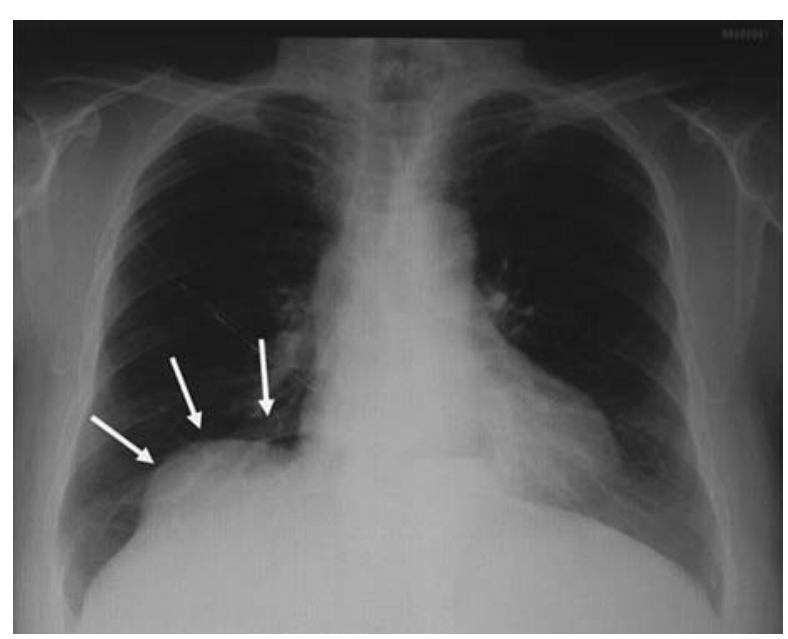

Figure 3. Photograph of the chest X-ray of the patient. The tumor protruding from the surface of the liver pushed the right diaphragm up toward the pleural cavity (arrows). the S8 region of the liver and had pushed the diaphragm up towards the pleural cavity (Fig. 3). In addition, laboratory data indicating hepatic reserve, such as serum levels of prothrombin time (PT), a hepaplastin test (HPT), chorine esterase (ChE), albumin (Alb), platelet (Plt), total cholesterol (T-cho) and total bilirubin (T-bil), were very poor: PT, 52\%; HPT, 40\%; ChE, 133 U/l; Alb, 2.8 g/dl; Plt, 6.6x104/ $/$ l; T-cho, $99 \mathrm{mg} / \mathrm{dl}$; and T-bil, $3.64 \mathrm{mg} / \mathrm{dl}$. Therefore, an extremely cautious treatment was highly desirable as not to impair the poor hepatic reserve.

Because the large-size tumor was hypervascular and was protruding from the surface of the liver, transcatheter arterial chemoembolization (TACE) was initially chosen to shut down the arterial blood supply. Abdominal angiography showed the massive tumor stain in the area of the right hepatic artery immediately under the diaphragm (Fig. 4). After injecting $30 \mathrm{mg}$ of epirubicin dissolved in $2 \mathrm{ml}$ of lipiodol into the tumor through the right hepatic artery, 


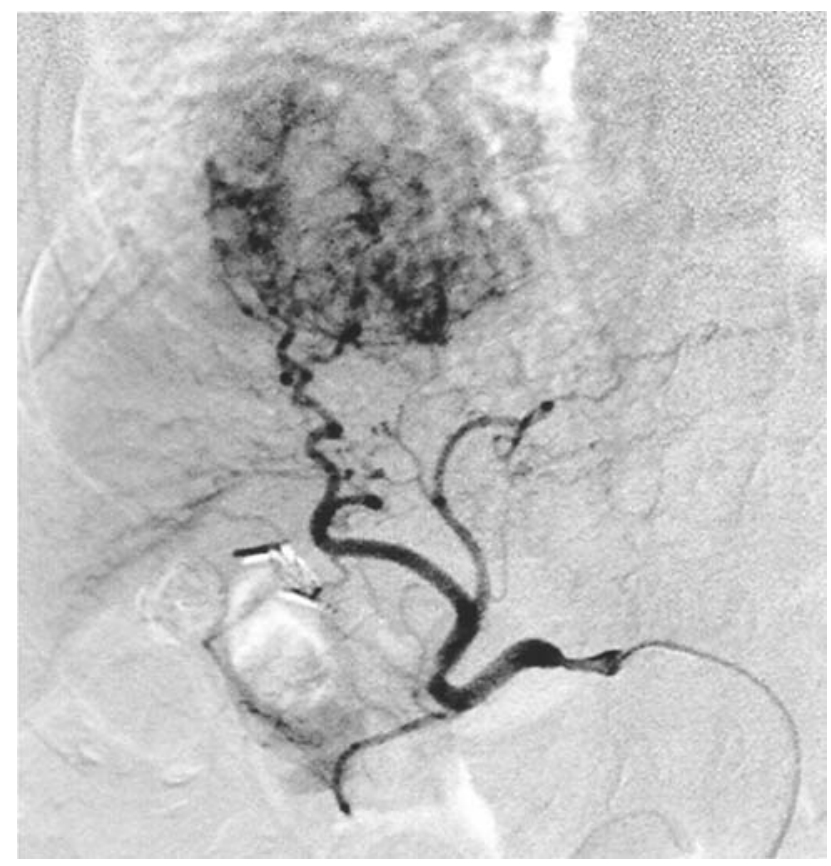

Figure 4. Angiography of the right hepatic artery. The massive tumor stain is shown directly under the diaphragm by right hepatic arteriography.

embolization of the main branch of the feeder artery was performed using pieces of gelfoam. Although the accumulation of lipiodol in the tumor was shown by CT 7 days after the angiography, the deposit of lipiodol in the tumor was loose, indicating that the tumor was not completely destroyed (Fig. 5). Severe hepatocellular damage and impairment of hepatic reserve fortunately did not occur after a single TACE treatment. Therefore, PELI-RFA and PELI, originally developed in our department, were additionally performed. PELI-RFA was performed twice for the large-size tumor and once for the daughter nodule at a relatively low power output for a short-time duration by using the Cool-tip RF system (Radionics, Burlington, VT, USA). For the large-size tumor, RFA was performed at $30 \mathrm{~W}$ for $2 \mathrm{~min}, 50 \mathrm{~W}$ for $2 \mathrm{~min}$ and $70 \mathrm{~W}$ for $2 \mathrm{~min}$ (6 min in total), after injecting $11 \mathrm{ml}$ of $15 \%$

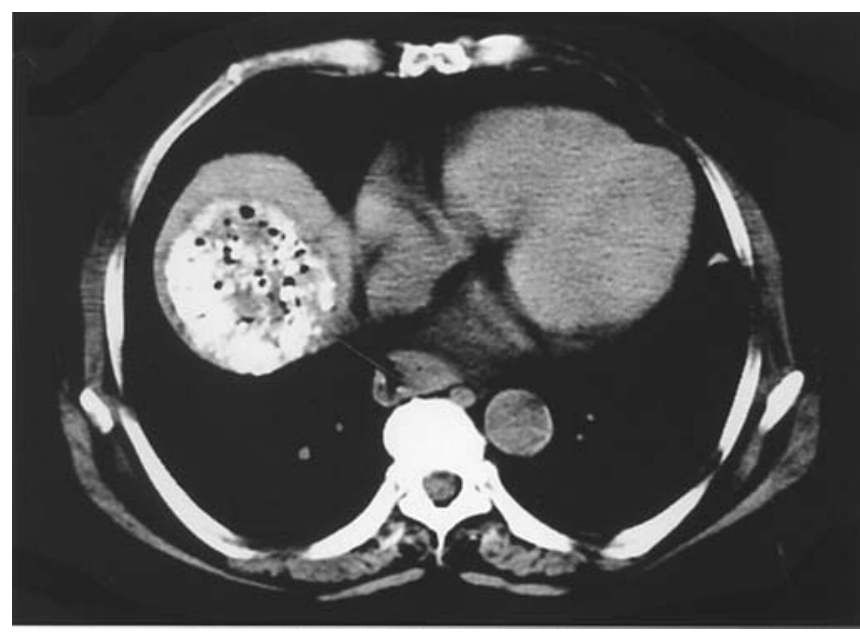

Figure 5. Photograph of plain CT of the large-size tumor in the S8 region of the liver 7 days after a single TACE treatment. In the TACE treatment, $30 \mathrm{mg}$ of epirubicin dissolved in $2 \mathrm{ml}$ of lipiodol was injected into the tumor through the right hepatic artery. The loose deposit of lipiodol in the tumor can be seen.

lipiodol in ethanol for the first RFA; and at $30 \mathrm{~W}$ for $2 \mathrm{~min}$, $50 \mathrm{~W}$ for $2 \mathrm{~min}, 70 \mathrm{~W}$ for $2 \mathrm{~min}$ and $80 \mathrm{~W}$ for $3 \mathrm{~min}(9 \mathrm{~min}$ in total), after injecting $10 \mathrm{ml}$ of $15 \%$ lipiodol in ethanol for the second RFA. In both PELI-RFA treatments, the final temperature achieved by the electrode was $70^{\circ} \mathrm{C}$. Since liver function was kept stable after PELI-RFA, PELI was further performed once 10 days after the second PELI-RFA. Eight milliliters of $15 \%$ lipiodol in ethanol was injected into the main tumor. In total, $29 \mathrm{ml}$ of $15 \%$ lipiodol in ethanol was injected into the main tumor throughout a series of PELIRFA and PELI. For the daughter nodule, RFA was performed at $30 \mathrm{~W}$ for $2 \mathrm{~min}$ and $60 \mathrm{~W}$ for $3 \mathrm{~min}$ after injecting $4.8 \mathrm{ml}$ of $15 \%$ lipiodol in ethanol. In the previous report, we stated that the therapeutic effects of PELI-RFA and PELI were easily and roughly evaluated by plain CT without using helical dynamic CT. Plain CT taken after PELI showed the homogeneously tight deposit of lipiodol in the whole tumor. Moreover, helical dynamic CT showed no enhancement of the lesions in the early vascular phase and no defect in the late

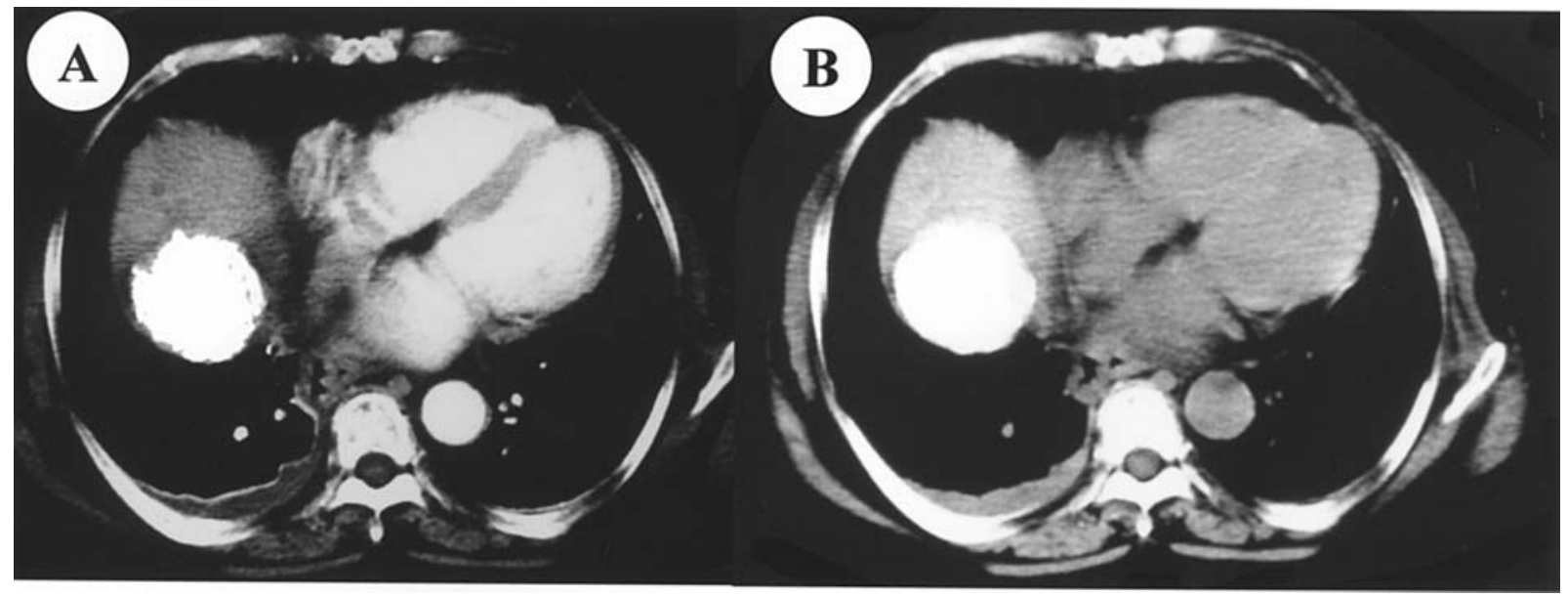

Figure 6. Photograph of helical dynamic CT of the large-size tumor in the S8 region of the liver after PELI-RFA and PELI. CT images of the early vascular phase (A) and the late phase (B) are shown in the figure. Lipiodol is homogeneously accumulated in the entire region of the tumor and no defect around the tumor is detected. 
phase, indicating that the entire tumor was destroyed (Fig. 6). Laboratory data indicating hepatic reserve were unchanged after treatment; PT, 47\%; HPT, 39\%; ChE, $153 \mathrm{U} / 1$; Alb, $2.9 \mathrm{~g} / \mathrm{dl}$; and T-bil, $3.1 \mathrm{mg} / \mathrm{dl}$. The elevated serum level of AFP was immediately decreased to an almost normal level (27 ng/ml), and that of PIVKA II was decreased to a normal level $(8 \mathrm{MAU} / \mathrm{ml})$. The patient felt good without major complaints throughout the admission period.

\section{Discussion}

HCC is one of the most common malignancies worldwide (1), especially in Asia. Despite the advances in diagnostic and therapeutic modalities for the treatment, HCC often progresses to an advanced stage. RFA was recently innovated for the treatment of HCC and is widely available for patients and clinicians worldwide at present (2-4). This newly developed technique appears very effective to induce wider coagulated necrosis than PEI without major complications $(10,11)$, but the efficacy of RFA is limited to relatively smallsize HCC with the maximum diameter $<3 \mathrm{~cm}$. Therefore, we have developed PEI-RFA for inducing wider coagulated necrosis $(12,13)$ and for achieving safer treatment of HCC (6). Furthermore, as modifications of PEI and PEI-RFA, we developed the treatment of injection of a mixture of ethanol and lipiodol instead of ethanol alone, PELI and PELI-RFA $(8,9)$. PELI was initially performed on an HCC patient with severe hepatic reserve, and we reported that PELI might be a milder treatment modality especially for patients with poor hepatic reserve (8). We reported that lipiodol dissolved in ethanol had a tight affinity to HCC cells. Indeed, a loose deposit of lipiodol in the tumor after a single TACE was changed to a tight deposit after PELI-RFA and PELI. Therefore, the combined use of lipiodol and ethanol may effectively destroy even large-size HCC.

Recently, we reported a case of advanced HCC successfully treated with the combined administration of 5-fluorouracil and pegylated IFN (14). The HCC in this case was diffusely distributed in the liver, and tumor thrombus had already developed in the major branches of the portal vein. Therefore, local treatment was not an appropriate approach. However, in the present case, the large-size HCC was an encapsulated lump of tumor, and the hepatic reserve of the patient was severely poor. Therefore, we judged that repeated administration of anticancer chemical reagents might affect the poor hepatic reserve so local treatment was suitable for avoiding this impairment. We carefully treated the patient by monitoring the changes in laboratory data. Laboratory data indicating hepatic reserve, such as PT, HPT, ChE, Alb, T-cho and T-bil, were unchanged and the general condition of the patient was continually stable during the series of treatments of TACE, PELI-RFA and PELI. Nevertheless, the tumor was totally destroyed and the tumor markers were decreased to almost normal levels. This successful case indicates that the combination therapy of PELI-RFA and PELI is a treatment modality that can safely treat large-size HCC without affecting hepatic reserve. Thus, the present case showing a complete response to the combination therapy provides us with insight into a promising approach for treating large-size advanced $\mathrm{HCC}$ of patients with poor hepatic reserve.

\section{Acknowledgements}

We are grateful to Ms. Aya Iwai, a clerk at KKR Takamatsu Hospital, for her technical support towards the completion of the manuscript.

\section{References}

1. Okuda K: Hepatocellular carcinoma. J Hepatol 32: 225-237, 2000.

2. Curley SA, Izzo F, Ellis LM and Nicholas Vauthey J: Radiofrequency ablation of hepatocellular cancer in 110 patients with cirrhosis. Ann Surg 232: 381-391, 2000.

3. Livraghi T, Goldberg SN, Lazzaroni S, Meloni F, Ierace T, Solbiati L and Gazelle GS: Hepatocellular carcinoma: radiofrequency ablation of medium and large lesions. Radiology 214 : 761-768, 2000.

4. Goldberg SN, Gazelle GS, Comon CC, Mueller PR and Tanabe KK: Treatment of intrahepatic malignancy with radiofrequency ablation. Cancer 88: 2452-2463, 2000.

5. Abdalla EK, Vauthey JN, Ellis LM, Ellis V, Pollock R, Broglio KR, Hess K and Curley SA: Recurrence and outcomes following hepatic resection, radiofrequency ablation, and combined resection/ablation for colorectal liver metastases. Ann Surg 239: 818-825, 2004.

6. Kurokohchi K, Watanabe S, Masaki T, Hosomi N, Miyauchi Y, Himoto T, Kimura Y, Nakai S, Deguchi A, Yoneyama H, Yoshida S and Kuriyama S: Comparison between combination therapy of percutaneous ethanol injection and radiofrequency ablation and radiofrequency ablation alone for patients with hepatocellular carcinoma. World J Gastroenterol 11: 1426-1432, 2005.

7. Kurokohchi K, Masaki T, Watanabe S, Nakai S, Deguchi A, Morishita A, Yoneyama H, Ohgi T, Ono M, Yoshitake A, Kako T, Ohmachi N, Kiuchi T, Maeta T, Yoshida M, Mori Y, Kohi F and Kuriyama S: Time-lag performance of radiofrequency ablation after percutaneous ethanol injection for the treatment of hepatocellular carcinoma. Int J Oncol 28: 971-976, 2006.

8. Kurokohchi K, Masaki T, Miyauchi Y, Funaki T, Yoneyama H, Miyoshi H, Yoshida S, Himoto T, Morishita A, Uchida N, Watanabe S and Kuriyama S: Percutaneous ethanol and lipiodol injection therapy for hepatocellular carcinoma. Int J Oncol 24: 381-387, 2004.

9. Kurokokohchi K, Masaki T, Miyauchi Y, Hosomi N, Yoneyama H, Yoshida S, Himoto T, Deguchi A, Nakai S, Inoue H, Watanabe S and Kuriyama S: Efficacy of combination therapies of percutaneous or laparoscopic ethanol-lipiodol injection and radiofrequency ablation. Int J Oncol 25: 1737-1743, 2004.

10. Lencioni RA, Allgaier H-P, Cloni D, Olschewski M, Deibert P, Crocetti L, Frings H, Laubenberger J, Zuber I, Blum HE and Bartolozzi C: Small hepatocellular carcinoma in cirrhosis: randomized comparison of radio-frequency thermal ablation versus percutaneous ethanol injection. Radiology 228: 235-240, 2003.

11. Livraghi T, Goldberg SN, Lazzaroni S, Meloni F, Solbiati L and Gazelle GS: Small hepatocellular carcinoma: treatment with radio-frequency ablation versus ethanol injection. Radiology 210: 655-661, 1999.

12. Kurokohchi K, Watanabe S, Masaki T, Hosomi N, Funaki T, Arima K, Yoshida S, Miyauchi Y and Kuriyama S: Combined use of percutaneous ethanol injection and radiofrequency ablation for the effective treatment of hepatocellular carcinoma. Int J Oncol 21: 841-846, 2002.

13. Kurokohchi K, Watanabe S, Masaki T, Hosomi N, Funaki T, Arima K, Yoshida S, Nakai S, Murota M, Miyauchi Y and Kuriyama S: Combination therapy of percutaneous ethanol injection and radiofrequency ablation against hepatocellular carcinomas difficult to treat. Int J Oncol 21: 611-615, 2002.

14. Kurokohchi K, Takaguchi K, Kita K, Masaki T and Kuriyama S: Successful treatment of advanced hepatocellular carcinoma by combined administration of 5-fluorouracil and pegylated interferon- $\alpha$. World J Gastroenterol 11: 5401-5403, 2005 